$\mathbb{T}$ periodica polytechnica

Electrical Engineering and Computer Science

$57 / 3(2013) 83$ 97

doi: 10.3311/PPee.2102

http://periodicapolytechnica.org/ee

Creative Commons Attribution (1)

RESEARCH ARTICLE

\section{High precision GPS positioning with multiple receivers using carrier phase technique and sensor fusion}

\author{
László Kis / Béla Lantos
}

Received 2013-05-09, accepted 2013-11-12

\begin{abstract}
This paper presents a carrier phase differential GPS technique for vehicle navigation. The orientation and position of the vehicle can reliably be calculated by the proposed solutions. The developed methods are aided by inertial and magnetic sensors. They are designed for dead reckoning and to handle phase slip. Therefore these solutions can be applied when the set of the available satellites changes frequently. Extended Kalman filters perform state estimation. The paper also presents the low-cost hardware/software architecture of the navigation system. The effectiveness of the methods have been proven in real car and flight tests.
\end{abstract}

\section{Keywords}

differential GPS $\cdot$ carrier phase $\cdot$ state estimation $\cdot$ navigation

\section{Acknowledgement}

The work of L. Kis was supported by the European Union and the State of Hungary, co-financed by the European Social Fund in the framework of TÁMOP 4.2.4. A/1-11-1-2012-0001 'National Excellence Program'.

The work of B. Lantos was supported by the European Union and Hungary in the project TÁMOP-4.2.2.A-11/1/KONV-20120012: "Basic research for the development of hybrid and electric vehicles".

\section{László Kis}

Department of Control Engineering and Information Technology, Budapest University of Technology and Economics, Magyar tudósok krt. 2., H-1117 Budapest, Hungary e-mail: 1kis@iit.bme.hu

\section{Béla Lantos}

Research Centre of Vehicle Industry, Széchenyi István University, Egyetem tér 1., H-9026 Győr, Hungary

e-mail: lantos@iit.bme.hu

\section{Introduction}

The outdoor navigation of autonomous vehicles has attracted the focus of the researchers and engineers in the recent decades. A variety of sensors have been applied to determine the state of the moving vehicle. However, the most challenging problem is the numerous unknown characteristics of the outdoor environment, which influence navigation.

Unmanned outdoor vehicles need an orientation and position determination system. To perform precise maneuvers, e.g. to avoid obstacles or move among corridors, a subdecimeter precision in position and subdegree precision in orientation are required.

Several different types of navigation approaches have appeared. A typical one is the vision based navigation. This solution makes it possible to determine the position of the vehicle relative to an object which can be seen by the camera. Therefore, it is suitable for local navigation. Because of the variety of environment, even the best vision based solutions need some restrictions in the area of application. On the other hand, it is usually easy to determine the orientation of a vehicle.

An other common sensor in outdoor navigation is the GPS. This solution makes it possible to calculate the position of a vehicle in a global coordinate system. The disadvantage of simple GPS receiver based solution is that the high error of the calculated position makes it unsuitable for certain applications.

More precise position result can be obtained by using the carrier phase differential GPS technique. In this approach, at least two receivers should be used with carrier phase measurement option. The wavelength of the L1 carrier signal of the GPS system is about 19 centimeters. The phase-locked loops inside the receiver can measure the actual phase with less than one centimeter precision. Therefore, subdecimeter precision in positioning is possible.

Because of the high precision, this technique is very popular in some applications, for example in geodesy [18], in harvesting [2] or in aerial transportation [7].

The common property of these fields is that the open sky view for the GPS antennas is large. Therefore, in the time of operation, there are enough satellites in view for reliable position de- 
termination. However, in other applications, where the set of the usable satellites changes frequently or the number of these satellites can go under a limit, it is hard to present a reliable method.

Another challenge is the integer ambiguity problem [11], $[16],[8]$. Its mathematical form is described in Appendix 7.1. The typical methods for solving this problem are the LAMBDA [17] and the (modified) MLAMBDA [4].

There are some practical problems with the typical carrier phase measurement based positioning solutions:

1 They require the knowledge of the correlation matrix of the carrier phase measurements [17], [15].

2 The LAMBDA based methods need some minutes to produce reliable result if the set of visible satellites is changing [3].

3 The typical phase slip detection methods are not real-time, they apply post-processing methods [16].

The goal of this paper is to elaborate real-time solutions of the above problems within a low cost hardware/software architecture, namely

- presenting reliable integer ambiguity resolution algorithm which can run in real-time, using only single epoch measurement

- solving the phase slip problem in real-time

- quick handling of the change of the visible satellites

- improving dead reckoning capabilities in GPS denied areas

- presenting a multi-modal sensor fusion technique for improving the real-time characteristics of high-precision GPS positioning in high-dynamic vehicular applications.

Our method can be divided into two levels of the realtime framework. First, the orientation is determined using the onboard GPS receivers and the integer ambiguity resolution method is aided by the accelerometer and the magnetometer sensors. This solution is designed for situations where the set of visible satellites changes frequently.

The second level is the position determination. It involves a fixed GPS receiver on the ground as a reference station.

Both the orientation and the position calculations apply extended Kalman filters (EKF), which perform a sensor fusion based on measurements provided by the receivers and other sensors.

The structure of the paper is as follows. Section 2 gives a short introduction to the field of the carrier phase differential GPS based on [3], [8] and [11]. Section 3 describes the orientation estimation method of the moving vehicle. Section 4 introduces the position determination algorithm. Section 5 presents the extended Kalman filter based state estimation. Finally, Section 6 presents the hardware structure of the system and shows the experimental results. Appendix A and B describe the origin of the carrier phase observables and the integer ambiguity problem, respectively.

\section{Baseline geometry and differencing techniques}

The simplest arrangement of a differential GPS system consists of two GPS receivers. The geometry of the two receivers can be seen in Fig. 1] It shows the actual state of the carrier phase signal at the time epoch $t$. The two receivers are indexed with $r$ and $s$ and the index $i$ refers to the $i^{t h}$ satellite.

The carrier phase measurement output of a GPS receiver has usually two components for each satellite. The first component is an integer number $I C P_{r}^{i}(t)$ called integrated carrier phase. The second component is the actual phase measurement $\varphi_{r}^{i}(t)$. If the phase measurement is represented in wavelength unit then $\varphi_{r}^{i}(t)$ is a real number in the interval [0...1). The sum of $\varphi_{r}^{i}(t)+I C P_{r}^{i}(t)$ is the carrier cycle measurement $\phi_{r}^{i}(t)$, described in Appendix 7.1

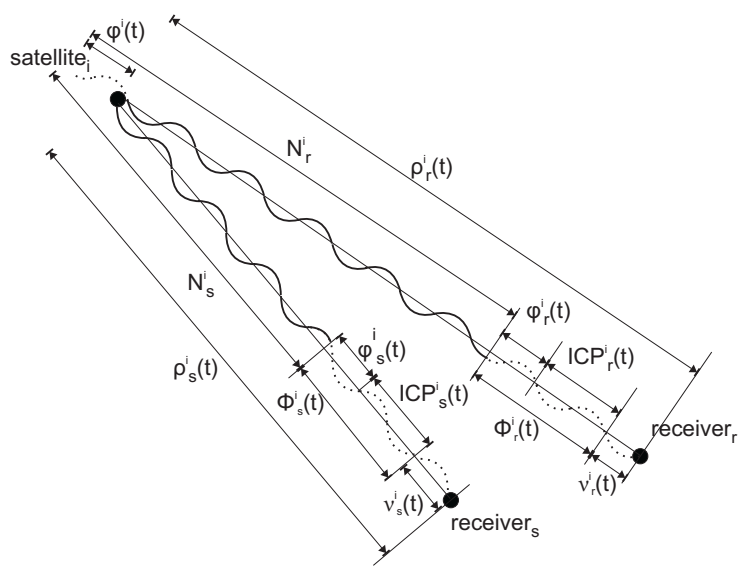

Fig. 1. The geometry of the differential GPS arrangement

Based on Fig. 1 and [11], the length of the propagation path $\rho_{r}^{i}(t)$ of the satellite signal between satellite $i$ and receiver $r$ is:

$$
\rho_{r}^{i}(t)=N_{r}^{i}-\varphi^{i}(t)+\phi_{r}^{i}(t)+v_{r}^{i}(t)
$$

where $N_{r}^{i}$ is an unknown integer number expressed in wavelength which is the sum of $\phi_{\text {init }, r}^{i}$ (see Appendix 7.1) and the number of carrier cycles between the satellite $i$ and receiver $r$ at time $t_{0} \cdot \varphi^{i}(t)$ is the transmitted satellite signal phase and $v_{r}^{i}(t)$ is the measurement error.

The following error model is introduced in [3], [6] and [8]:

$$
\begin{aligned}
v_{r}^{i}(t)= & I_{r}^{i}(t)-T_{r}^{i}(t)+c \lambda^{-1}\left(\delta t^{i}(t)-\delta t_{r}(t)\right) \\
& +c \lambda^{-1}\left(D_{r}(t)-D^{i}(t)\right)+\mu_{r}^{i}(t)
\end{aligned}
$$

where $I_{r}^{i}(t)$ and $T_{r}^{i}(t)$ are the ionospheric and tropospheric delays, $\delta t^{i}(t)$ and $\delta t_{r}(t)$ are the clock errors of the satellite and the receiver, $D^{i}(t)$ and $D_{r}(t)$ are the hardware delays of the satellite and the receiver. Constants $c$ and $\lambda$ are the speed of light and the wavelength of the carrier signal. Variable $\mu_{r}(t)$ is the measurement noise including the multipath effect. According to [3] $\mu_{r}(t)$ is normally distributed with zero expected value.

Similar interpretation yields for receiver s:

$$
\begin{aligned}
\rho_{s}^{i}(t)= & N_{s}^{i}-\varphi^{i}(t)+\phi_{s}^{i}(t)+v_{s}^{i}(t) \\
v_{s}^{i}(t)= & I_{s}^{i}(t)-T_{s}^{i}(t)+c \lambda^{-1}\left(\delta t^{i}(t)-\delta t_{s}(t)\right) \\
& +c \lambda^{-1}\left(D_{s}(t)-D^{i}(t)\right)+\mu_{s}^{i}(t)
\end{aligned}
$$


Let us define the following variables:

$$
\begin{aligned}
\Delta \rho^{i}(t) & =\rho_{r}^{i}(t)-\rho_{s}^{i}(t) \\
\Delta \phi^{i}(t) & =\phi_{r}^{i}(t)-\phi_{s}^{i}(t) \\
\Delta \mu^{i}(t) & =\mu_{r}^{i}(t)-\mu_{s}^{i}(t) \\
\beta(t) & =c \lambda^{-1}\left(\delta t_{s}(t)-\delta t_{r}(t)+D_{r}(t)-D_{s}(t)\right)
\end{aligned}
$$

If the two receivers are close to each other, it can be assumed that the tropospheric and the ionospheric error is the same for the two receivers [11]. Therefore

$$
v_{r}^{i}(t)-v_{s}^{i}(t)=\beta(t)+\Delta \mu^{i}(t)
$$

Subtracting (3) from (1) yields

$$
\Delta \phi^{i}(t)=\Delta \rho^{i}(t)-\Delta N^{i}-\beta(t)-\Delta \mu^{i}(t)
$$

The geometry of the two receiver differential GPS system can also be introduced using a different approach. This is based on the fact that the distance between the receiver and the satellite is significantly larger than the distance between the two receivers. It implies that the propagation directions of the radio signals received by the two receivers are approximately parallel to each other. This case is shown in Fig. 2

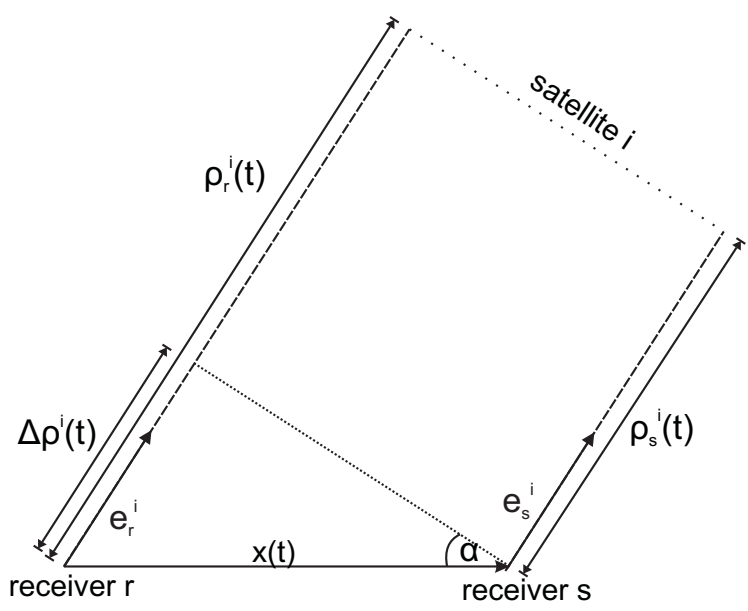

Fig. 2. Geometry of the two receivers

The direction unit vectors between the $i^{\text {th }}$ satellite and the receivers are denoted by $e_{r}^{i}(t)$ and $e_{s}^{i}(t)$, respectively. Let us define $e^{i}(t):=e_{r}^{i}(t) \approx e_{s}^{i}(t)$. The vector between the two receivers position is $x(t)$, called baseline. From the geometry of Fig. 2 one can write

$$
\Delta \rho^{i}(t)=\lambda^{-1} e^{i}(t)^{T} x(t)
$$

The satellite direction $e^{i}$ can be calculated using the data transmitted by the satellite. In the first step the position of the satellite should be determined using the transmitted ephemeris data and the algorithm described in [1]. The result will be in the Earth centered Earth fixed (ECEF) coordinate frame. In the next step the rough user position is required. Most of the receivers are able to produce the rough user position in ECEF. If the receivers produce this information only in longitude-latitudealtitude form, the transformation of [6] can be used. As the accuracy of the satellite and user position calculation are in the range of some meters and the nominal altitude of the satellite is $20200 \mathrm{~km}$ [6], the result for $e^{i}$ is precise enough for further algorithms.

At the end of the calculation $e^{i}$ is described in the ECEF coordinate frame. By substituting (11) into (10), the so called single difference equation can be formed:

$$
\Delta \phi^{i}(t)=\lambda^{-1} e^{i}(t)^{T} x(t)-\Delta N^{i}-\beta(t)-\Delta \mu^{i}(t)
$$

Equation (12) has a satellite independent $\beta(t)$ variable. There are solutions which can deal with this variable, like in [3], but the typical solution to eliminate this unknown variable is the usage of the double differencing technique [7], [11].

Let the following double differences be defined for satellite $i$ and $j$ as

$$
\begin{aligned}
\nabla \Delta \phi^{i, j}(t) & =\Delta \phi^{i}(t)-\Delta \phi^{j}(t) \\
\nabla \Delta N^{i, j} & =\Delta N^{i}-\Delta N^{j} \\
\nabla \Delta \mu^{i, j}(t) & =\Delta \mu^{i}(t)-\Delta \mu^{j}(t) \\
E^{i, j}(t) & =e^{i}(t)-e^{j}(t)
\end{aligned}
$$

Forming (12) for satellite $i$ and satellite $j$ and subtracting the two equations from each other, the double differenced equation is derived

$$
\nabla \Delta \phi^{i, j}(t)=\lambda^{-1} E^{i, j}(t)^{T} x(t)-\nabla \Delta N^{i, j}-\nabla \Delta \mu^{i, j}(t)
$$

Without the loss of generality, let a master satellite be indexed by 1 . The number of satellites in use is denoted by $m$. The following notations for the $k^{\text {th }}$ measurement epoch will be introduced:

$$
\begin{aligned}
& \nabla \Delta \phi_{k}=\left[\begin{array}{c}
\nabla \Delta \phi^{1,2}\left(t_{k}\right) \\
\vdots \\
\nabla \Delta \phi^{1, m}\left(t_{k}\right)
\end{array}\right] \quad E_{k}=\left[\begin{array}{c}
E^{1,2}\left(t_{k}\right)^{T} \\
\vdots \\
E^{1, m}\left(t_{k}\right)^{T}
\end{array}\right] \\
& \nabla \Delta N=\left[\begin{array}{c}
\nabla \Delta N^{1,2} \\
\vdots \\
\nabla \Delta N^{1, m}
\end{array}\right] \quad \nabla \Delta \mu_{k}=\left[\begin{array}{c}
\nabla \Delta \mu^{1,2}\left(t_{k}\right) \\
\vdots \\
\nabla \Delta \mu^{1, m}\left(t_{k}\right)
\end{array}\right]
\end{aligned}
$$

One can formulate the problem as

$$
\nabla \Delta \phi_{k}=\lambda^{-1} E_{k} x_{k}-\nabla \Delta N-\nabla \Delta \mu_{k}
$$

The left-hand side of 18 can be formed from the measurement of the GPS receivers. On the right-hand side of the equation are the unknown baseline $x$ and the time independent double differenced integer ambiguity $\nabla \Delta N$. According to [3] and [8] the elements of the measurement noise vector $\nabla \Delta \mu_{k}$ are normally distributed and correlated. In a practical case the covariance matrix of $\nabla \Delta \mu_{k}$ is hard to define only from the measurements of $\nabla \Delta \phi_{k}$. Therefore methods [5], [15], [17] which require this covariance matrix usually produce false positive result from a single epoch measurement. 


\section{Orientation estimation with three antenna system}

The number of unknown parameters in 18 is $m+2$, namely three components of $x$ and $m-1$ components of $\nabla \Delta N$, but the number of linearly independent equations is only $m-1$. Therefore, additional information is needed so that $(18)$ can be solved.

One of this additional details can be the integer property of $\nabla \Delta N^{i, j}$. The classical solutions, like LAMBDA method [17] or MILES [5] rely on this property only.

In some special cases additional sensors (like inertial or magnetic sensors) are present, which can give information to make the result more reliable. The goal of this section is the determination of the orientation of a vehicle using carrier phase GPS measurements. In vehicle navigation there can be additional sensors on the board. Our method will use the magnetometer and the accelerometer for the initialization of the integer ambiguity resolution. The angular velocity sensor will be used for the detection of the phase slip problem.

The GPS antennas will be mounted and fixed on the vehicle, therefore their positions relative to each other are fixed. Hence the lengths of the vectors between the antennas are also known constraints.

The arrangement of the sensors and coordinate systems can be seen in Fig. 3. In this example the vehicle is a car, but it could be any other type of moving vehicle.

The vehicle movement is assumed to be a short range movement, therefore the reference coordinate frame is chosen as the local North-East-Down (NED) coordinate system.

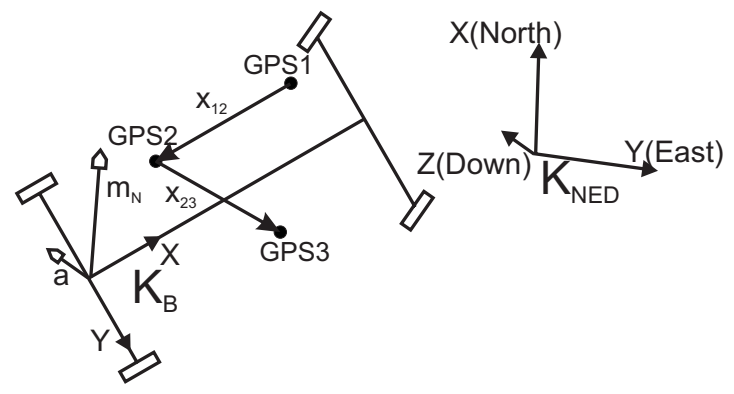

Fig. 3. The three antenna structure

\subsection{Initialization of the integer ambiguity determination}

In Fig. 3 the positions of the three GPS antennas are denoted by $p_{1, B}, p_{2, B}, p_{3, B}$ and are considered to be known in the body frame $K_{B}$. Hence the vectors between the antennas in $K_{B}$ are also known as $x_{12, B}=p_{1, B}-p_{2, B}$ and $x_{23, B}=p_{2, B}-p_{3, B}$.

The north direction is measured by the magnetometer and is denoted by $m_{N}$. It is assumed that the magnetometer is calibrated and the result is corrected with the inclination and declination angle of the local magnetic field. One possible solution is described in [13].

Let $a$ be the measurement of the accelerometer in the position of the origin of $K_{B}$. It is also assumed that the accelerometer is calibrated. In a stationary (not accelerating) situation, the measured vector is the gravitational acceleration directing to the up direction of $K_{N E D}$.

An approximation for the rotation between $K_{N E D}$ and $K_{B}$ can be given by

$$
\hat{R}_{B, N E D}=\left[\begin{array}{lll}
\frac{m}{\|m\|} & \frac{m \times a}{\|m \times a\|} & -\frac{a}{\|a\|}
\end{array}\right]
$$

hence an approximation for $x_{12}$ and $x_{23}$ in $K_{N E D}$ are

$$
\begin{aligned}
& \hat{x}_{12, N E D}=\hat{R}_{B, N E D}^{T} x_{12, B} \\
& \hat{x}_{23, N E D}=\hat{R}_{B, N E D}^{T} x_{23, B}
\end{aligned}
$$

where index $B$ refers to $K_{B}$ and

$$
\begin{aligned}
& \hat{x}_{12, E C E F}=R_{E C E F, N E D} \hat{x}_{12, N E D} \\
& \hat{x}_{23, E C E F}=R_{E C E F, N E D} \hat{x}_{23, N E D}
\end{aligned}
$$

This means that the initial guess for the double differenced integer ambiguity $\nabla \Delta N$ at the $k^{\text {th }}$ epoch can be

$$
\begin{aligned}
& \nabla \Delta \hat{N}_{12}=\nabla \Delta \phi_{12, k}-\lambda^{-1} E_{12, k} \hat{x}_{12, E C E F, k} \\
& \nabla \Delta \hat{N}_{23}=\nabla \Delta \phi_{23, k}-\lambda^{-1} E_{23, k} \hat{x}_{23, E C E F, k}
\end{aligned}
$$

\subsection{Determination of $R_{B, N E D}$}

At this point the task is to determine the $R_{B, N E D}$ rotation matrix using the $x_{12}$ and $x_{23}$ vectors measured in the NED coordinate frame. Let's introduce the normalized vectors in the form of

$$
\begin{aligned}
\bar{x}_{12, B} & =\frac{x_{12, B}}{\left\|x_{12, B}\right\|} \\
\bar{x}_{23, B} & =\frac{x_{23, B}}{\left\|x_{23, B}\right\|} \\
\bar{x}_{12, N E D} & =\frac{x_{12, N E D}}{\left\|x_{12, N E D}\right\|} \\
\bar{x}_{23, N E D} & =\frac{x_{23, N E D}}{\left\|x_{23, N E D}\right\|}
\end{aligned}
$$

The goal is to find the rotation $R_{B, N E D}$ which satisfy the equations

$$
\begin{aligned}
& \bar{x}_{12, B}=R_{B, N E D} \bar{x}_{12, N E D} \\
& \bar{x}_{23, B}=R_{B, N E D} \bar{x}_{23, N E D}
\end{aligned}
$$

Since $\bar{x}_{12, B}, \bar{x}_{23, B}, \bar{x}_{12, N E D}$ and $\bar{x}_{23, N E D}$ vectors have unit length, the system of equations has four independent equations. But $R_{B, N E D}$ has only three degrees of freedom, therefore the problem is overdefinite. Let the angle between $\bar{x}_{12, N E D}$ and $\bar{x}_{23, N E D}$ vectors be denoted by $\alpha_{N E D}$ and the angle between $\bar{x}_{12, B}$ and $\bar{x}_{23, B}$ be $\alpha_{B}$

$$
\begin{aligned}
\alpha_{B} & =\operatorname{acos}\left(\bar{x}_{12, B}^{T} \bar{x}_{23, B}\right) \\
\alpha_{N E D} & =\operatorname{acos}\left(\bar{x}_{12, N E D}^{T} \bar{x}_{23, N E D}\right) \\
\alpha_{N E D} & \approx \alpha_{B}
\end{aligned}
$$

On the other hand, rotation $R_{B, N E D}$ keeps the angle between the vectors. Hence only a suboptimal solution exists for $R_{B, N E D}$. 
Let $R_{B, N E D}$ be represented by three independent parameters $\varphi, \vartheta$, $\psi$ called roll-pitch-yaw angles. Let $\epsilon_{R}$ be used as an error vector defined by

$$
\epsilon_{R}=\left[\begin{array}{l}
R_{B, N E D}(\varphi, \vartheta, \psi) \bar{x}_{12, N E D}-\bar{x}_{12, B} \\
R_{B, N E D}(\varphi, \vartheta, \psi) \bar{x}_{23, N E D}-\bar{x}_{23, B}
\end{array}\right]
$$

which is a non-linear relationship. To solve this problem numeric optimization methods can be used, the authors used the Levenberg-Marquard method.

\subsection{Searching for the integer ambiguity}

In this subsection two possible solutions are shown to determine the proper $\nabla \Delta N$.

The first method uses the random sample consensus (RANSAC) approach [9]. The method neglects one or more linear equations from (24) and determine the double differenced integer ambiguity. Then it repeats the ambiguity determination by neglecting a different equation:

$$
\begin{aligned}
& {\left[\begin{array}{c}
\nabla \Delta N^{1,2} \\
\vdots \\
\nabla \Delta N^{1, i-1} \\
\nabla \Delta N^{1, i+1} \\
\vdots \\
\nabla \Delta N^{1, m}
\end{array}\right]=} \\
& {\left[\begin{array}{c}
\nabla \Delta \phi_{k}^{1,2} \\
\vdots \\
\nabla \Delta \phi_{k}^{1, i-1} \\
\nabla \Delta \phi_{k}^{1, i+1} \\
\vdots \\
\nabla \Delta \phi_{k}^{1, m}
\end{array}\right]-\lambda^{-1}\left[\begin{array}{c}
E_{k}^{1,2}{ }^{1, i-1}{ }_{k} \\
\vdots \\
E^{1, i+1} T \\
\vdots \\
\vdots \\
E_{k}^{1, m}{ }_{k}
\end{array}\right] \hat{x}_{12, E C E F, k}}
\end{aligned}
$$

where the outer brackets on the right side mean the round operation applied on the components of the real vector.

In this way different solutions are given for $\nabla \Delta N^{1, i}$. Then that value is chosen for the proper ambiguity which has the most frequent occurrence.

The second method starts from the $\nabla \Delta \hat{N}_{12}$ in 24). First a search space is defined as

$$
\left\lfloor\nabla \Delta \hat{N}_{12}^{1, i}-N_{\text {thres }}\right\rfloor \leq \nabla \Delta \hat{N}_{12}^{1, i} \leq\left\lceil\nabla \Delta \hat{N}_{12}^{1, i}+N_{\text {thres }}\right\rceil
$$

where $N_{\text {thres }}$ can be chosen based on the standard deviation of the magnetometer and the accelerometer, but in practice in most cases $N_{\text {thres }}=1$ is satisfactory. By this way the search space is not too large, hence all possibilities can be tested in real-time. The test criteria is

$$
\begin{aligned}
& \nabla \Delta N_{12}= \\
& \quad \operatorname{argmin}\left\|\left(\lambda E^{\dagger}\left(\nabla \Delta \phi_{12, k}-\nabla \Delta N_{12, j}\right)\right)-\hat{x}_{12, E C E F, k}\right\|
\end{aligned}
$$

where the minimum is calculated for every $\nabla \Delta N_{12, j}$ in the search space and $E^{\dagger}$ denotes Moore-Penrose pseudoinverse.
In practice the two methods can be combined. First the RANSAC based method is used. In this way a significantly false carrier phase measurement can be filtered out. In the second step the search space based method is used, where the center of the search space is the result of the RANSAC method.

\subsection{Handling the phase slip problem}

The phase slip occurs as a measurement error between two consecutive measurement epochs. In this case the GPS receiver hardware cannot detect one or more zero crosses in the GPS signal. This means that the carrier cycle measurement for the given satellite will be incorrect and the difference between the correct and the measured value will be an integer number of a half cycle.

Most of the GPS receivers, which are able to measure carrier phase, have the function to indicate the possibility of the phase slip, but the amount of the phase slip error is not given by the receiver. The following algorithm is recommended to determine the value of the phase slip error, using the angular velocity sensor on the moving vehicle.

Let (18) be described for two consecutive measurement epochs.

$$
\begin{aligned}
\nabla \Delta \phi_{k-1} & =\lambda^{-1} E_{k-1} x_{k-1}-\nabla \Delta N-\nabla \Delta \mu_{k-1} \\
\nabla \Delta \phi_{k} & =\lambda^{-1} E_{k} x_{k}-\nabla \Delta N-\nabla \Delta \mu_{k}
\end{aligned}
$$

Let us introduce

$$
\delta \nabla \Delta \phi_{k}=\nabla \Delta \phi_{k}-\nabla \Delta \phi_{k-1}
$$

and use the fact that for consecutive epochs $E_{k-1} \approx E_{k}$ and that $\mu_{k}$ and $\mu_{k-1}$ have the same noise parameters. Subtracting 39. from 40 , the result is

$$
\delta \nabla \Delta \phi_{k}=\lambda^{-1} E_{k} \Delta x_{k}-2 \nabla \Delta \mu_{k}
$$

where $\Delta x_{k}=x_{k}-x_{k-1}$

The $\Delta x_{k}$ value can be estimated using the measurement of the angular velocity sensor and the known value of $x_{k-1}$. The estimated $\Delta x_{k}$ is calculated as

$$
\Delta \hat{x}_{k, N E D}=\left(R_{B, N E D, k-1}^{T} R(\omega) R_{B, N E D, k-1}-I_{3}\right) x_{k-1, N E D}
$$

where $R_{B, N E D, k-1}$ is the rotation between $K_{B}$ and $K_{N E D}$ in the $(k-1)^{t h}$ epoch calculated from $x_{12, N E D, k-1}$ and $x_{23, N E D, k-1}$, while $R(\omega)$ is the Rodrigues rotation calculated from the measurements of the angular velocity sensor in the form of

$$
\begin{aligned}
t & =\frac{\omega}{\|\omega\|} \\
\alpha & =T_{s}\|\omega\| \\
R(\omega) & =C_{\alpha} I_{3}+\left(1-C_{\alpha}\right) t t^{T}+S_{\alpha}[t \times]
\end{aligned}
$$

where $\omega$ is the angular velocity measured in $K_{B}$ and $T_{s}$ is the sampling time of the angular velocity measurement. Then an error value can be formed as

$$
\epsilon_{k}=\delta \nabla \Delta \phi_{k}-\lambda^{-1} E_{k} \Delta \hat{x}_{k}
$$


If the double differenced carrier phase measurement noise $\mu_{k}$ is smaller than a quarter of the wavelength, then a phase slip will cause a significant increase or decrease in $\epsilon_{k}$, and a good approximation for the amount of the cycle slip value is the rounded value of $\epsilon_{k}$ towards an integer multiple of half cycle $(=0.5 K, \quad K \in \mathbb{Z})$.

\subsection{Solution for varying satellite sets}

In order to use the baseline determination methods, the disappearance of some used satellites and the appearance of some new satellites should be handled. This problem implies some challenges.

To form $\nabla \Delta N$, a master satellite is also needed. After the initialization the index of the master satellite is known from the previous measurement epoch. However it could happen, that the previous master satellite is not available in the current epoch.

It is also possible that only a few satellites are available, such that even with a given double differenced integer ambiguity, (18) cannot be solved. A recommended solution is to estimate the baseline using additional sensors.

When a new satellite appears it should be included into the set of the used satellites. In this case the new double differenced integer ambiguity should be found regarding the new satellite.

The flow chart of the orientation determination can be seen in Fig. 4

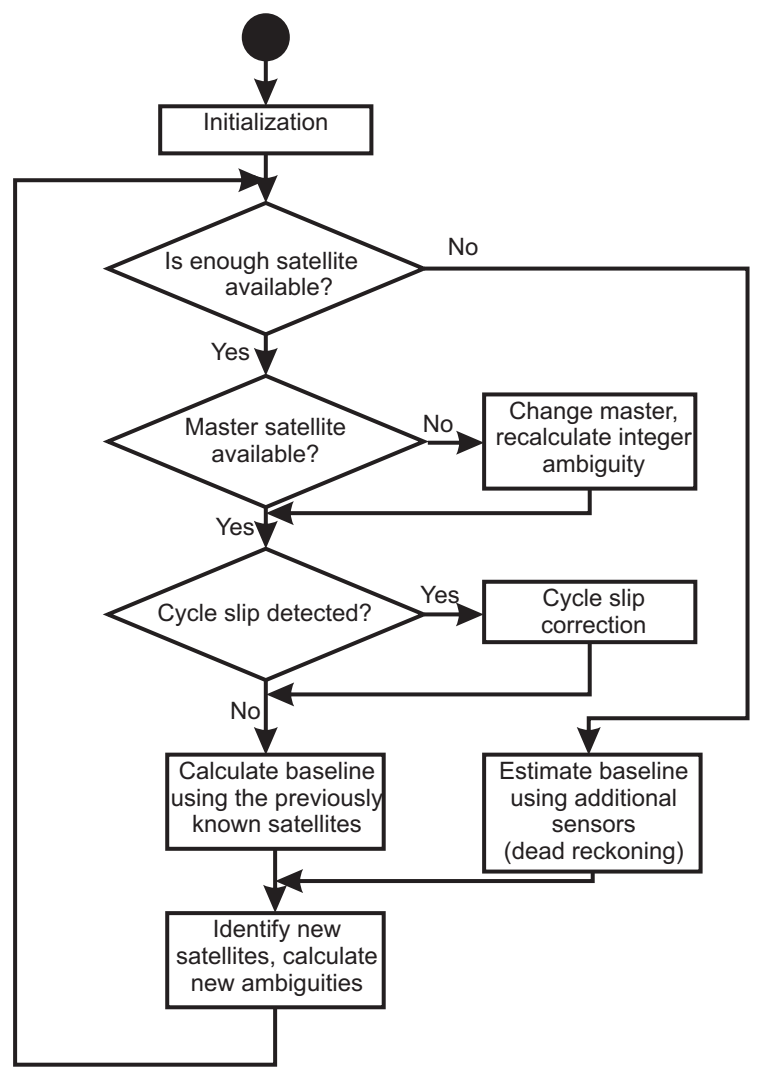

Fig. 4. Flowchart of the baseline determination

\subsubsection{Determining satellite availability}

The answer for the question "is there enough satellite for $x_{12}$ and $x_{23}$ determination" depends on whether (18) can be solved when $\nabla \Delta N$ is given. Two requirements should be met.

Requirement 1 There are three unknown variables in the baseline, therefore at least four satellites are needed.

Requirement 2 For the determination of $x$, the inverse or the pseudo-inverse of $E_{k}$ should exist and its numeric value should be determined precisely. Therefore if the number of the available satellites is higher than four the condition number of $E_{k}$ (i.e. the quotient of maximum and minimum singular values) should also be under a limit.

\subsubsection{Change the master satellite}

Let $\nabla \Delta N$ be given from the $(k-1)^{\text {th }}$ epoch and the master satellite is the $i^{t h}$ one. This master satellite will disappear in the next epoch, therefore $\nabla \Delta N$ should be recalculated. Let satellite $j$ be the new master satellite. It comes from (14), that

$$
\nabla \Delta N^{j, l}=\nabla \Delta N^{i, l}-\nabla \Delta N^{i, j}
$$

therefore every component of the new $\nabla \Delta N$ can be calculated.

\subsubsection{Handle new satellites}

Let $m+1$ be the index of the new satellite. Then the task is to determine the double differenced integer ambiguity for the new satellite. An initial approximation can be the following:

$$
\nabla \Delta \hat{N}^{i, m+1}=\left[\nabla \Delta \phi^{i, m+1}-E^{i, m+1} \hat{x}\right]
$$

where $i$ is the index of the master satellite and $\hat{x}$ is the baseline calculated using other satellite measurements or using dead reckoning. The goal is to find the reliable initial ambiguity. The recommended method is to use the second method in Subsection 3.3 . In this case the integer ambiguity is already known for the first $m$ satellite. Therefore the search space of (37) is applied only for $\nabla \Delta \hat{N}^{i, m+1}$, thus the computation capacity requirement is much smaller.

\subsubsection{Baseline dead reckoning}

When $x_{12}$ and $x_{23}$ cannot be determined from the GPS measurement, the simplest solution is to estimate the baseline from the previous baseline by accumulating the measurements of the angular velocity sensor. The recursive equation is

$$
x_{k}=R_{B, N E D, k-1}^{T} R(\omega) R_{B, N E D, k-1} x_{k-1}
$$

A more intelligent solution is to handle the varying offset of the angular velocity sensor. This can be achieved by using an extended Kalman-filter. Let the following discrete nonlinear dynamic system be considered as

$$
\begin{aligned}
x_{12, N E D, k} & =R_{B, N E D, k-1}^{T} R\left(\omega-\omega_{b}\right) R_{B, N E D, k-1} x_{k-1}+v_{x} \\
\omega_{k} & =\omega_{k-1}+v_{b} \\
x_{12, N E D, k, \text { meas }} & =x_{12, N E D, k}+z_{\text {meas }, k}
\end{aligned}
$$


Defining the input $\left(u_{k}\right)$, output $\left(y_{k}\right)$, noise $\left(v_{k}\right)$ and state variables $\left(x_{k}\right)$ of the system as

$$
\begin{aligned}
x_{k} & =\left[\begin{array}{ll}
x_{12, N E D, k}^{T} & \omega_{b, k}^{T}
\end{array}\right]^{T} \\
u_{k} & =\omega_{k} \\
v_{k} & =\left[\begin{array}{ll}
v_{x}^{T} & v_{b}^{T}
\end{array}\right]^{T} \\
y_{k} & =x_{12, N E D, k, \text { meas }}
\end{aligned}
$$

then the system is in a compact form of

$$
\begin{aligned}
& x_{k}=f_{\text {ori }}\left(x_{k-1}, u_{k-1}, v_{k-1}\right) \\
& y_{k}=g_{\text {ori }}\left(x_{k}, z_{k}\right)
\end{aligned}
$$

and an extended Kalman-filter can be applied parallel to the determination of $x_{12}$ and $x_{23}$. The case when there are not enough GPS measurements for baseline determination can be handled by skipping the update step of the extended Kalman-filter. The estimation method is illustrated in Fig. 5

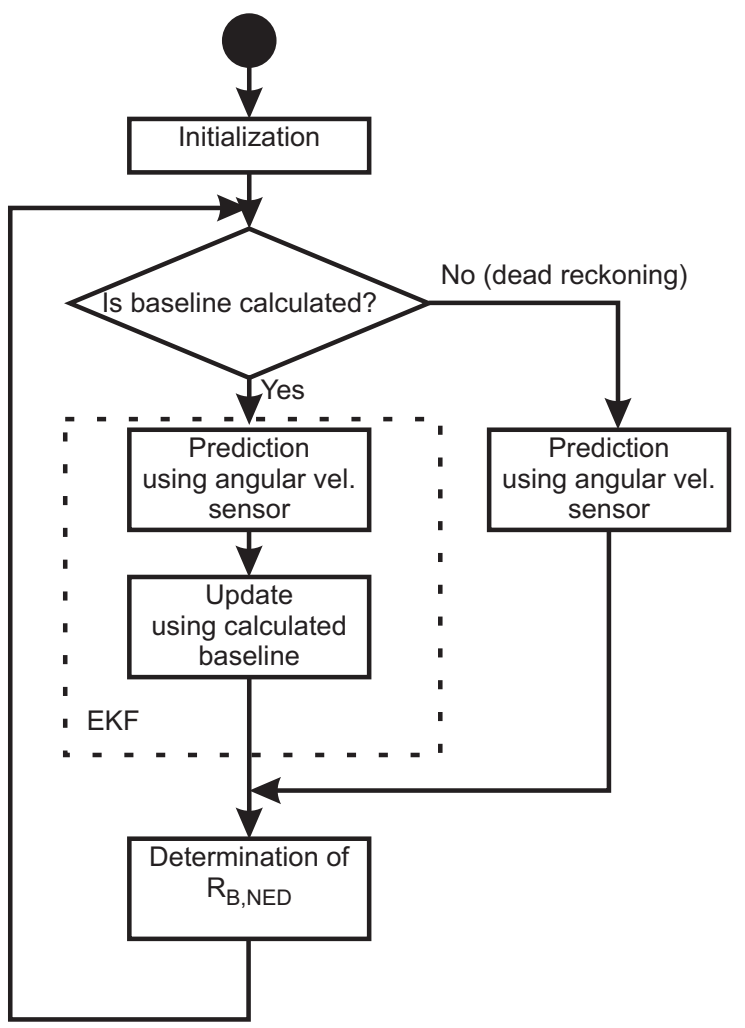

Fig. 5. Flow diagram of the baseline estimation

\section{Precise Position Determination}

As the orientation of the moving vehicle is already precisely known using carrier phase differential GPS technique, the next task is to determine the precise position of the vehicle. Keeping the differential approach, an additional GPS receiver should be introduced. This receiver is on the ground, its position is fixed and assumed to be precisely known and its name is base station. The goal of the algorithm is to determine the vector between the base station and one of the receivers on the moving object. The arrangement of the set of receivers can be seen in Fig. 6. In this task the actual values of $x_{12}$ and $x_{23}$ are assumed to be known in both the $K_{N E D}$ and $K_{B}$ frames.

The goal is to define the navigation information in the NorthEast-Down coordinate system. The property which makes the simplification in (9) possible is true if the receivers are close to each other. In practice, the high precision can be achieved when the moving receivers are not farther from the base station than approximately 50 kilometers. Therefore a practical choice can be that the origin of the local NED coordinate system is the position of the base station and the rotation $R_{E C E F, N E D}$ is calculated for the place of the base station.

\subsection{Usability of differential sensors}

The orientation computation uses the additional onboard sensors to strengthen the result of the carrier phase based orientation determination. It would be obvious to use these sensors in the position determination as well. Let us examine the capability of these sensors.

Because of the offset error of the inertial sensors, they can produce reliable information only about $\Delta x_{k}$ between consecutive measurement epochs. Using (42) and rearranging it for $\Delta x_{k}$ and assuming that $E_{k-1} \approx E_{k}$ it becomes

$$
\Delta x_{k}=\lambda\left(E_{k}\right)^{\dagger}\left(\delta \nabla \Delta \phi_{k}+2 \nabla \Delta \mu_{k}\right)
$$

The change of the position vector relative to the base station between an initial measurement epoch and the actual one is

$$
\Delta x_{0, k}=\lambda \sum_{i=1}^{k}\left(E_{i}\right)^{\dagger}\left(\delta \nabla \Delta \phi_{i}+2 \nabla \Delta \mu_{i}\right)
$$

These equations do not contain $\nabla \Delta N$, hence the information about the relative displacement has no addition to the double differenced integer ambiguity. Therefore the inertial sensors on the board of the vehicle cannot refine the carrier phase based measurements.

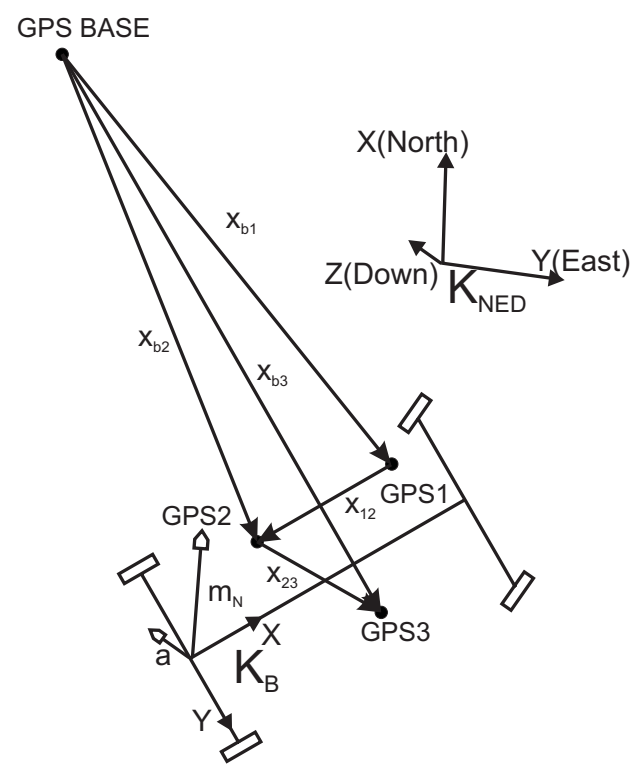

Fig. 6. Structure of the base station, moving receiver approach 


\subsection{Phase slip effect in position determination}

It is also important to examine the effect of the phase slip on $x_{b 1}, x_{b 2}$ and $x_{b 3}$. Let a phase slip be considered in the $j^{\text {th }}$ epoch. Then using (61) and 42) and some algebraic manipulations the relative displacement from the initial position yields

$$
\begin{aligned}
\Delta x_{0, k}= & \lambda \sum_{i=1}^{j-1}\left(E_{i}\right)^{\dagger}\left(\nabla \Delta \phi_{i}-\nabla \Delta \phi_{i-1}\right) \\
& +\lambda\left(E_{j}\right)^{\dagger}\left(\nabla \Delta \phi_{j}+\nabla \Delta \phi_{\epsilon}-\nabla \Delta \phi_{j-1}\right) \\
& +\lambda \sum_{i=j+1}^{k}\left(E_{i}\right)^{\dagger}\left(\nabla \Delta \phi_{i}+\nabla \Delta \phi_{\epsilon}-\nabla \Delta \phi_{i-1}-\nabla \Delta \phi_{\epsilon}\right) \\
& +\lambda \sum_{i=1}^{k}\left(E_{i}\right)^{\dagger} 2 \nabla \Delta \mu_{i} \\
= & \lambda \sum_{i=1}^{k}\left(E_{i}\right)^{\dagger}\left(\delta \nabla \Delta \phi_{i}+2 \nabla \Delta \mu_{i}\right)+\lambda\left(E_{j}\right)^{\dagger} \nabla \Delta \phi_{\epsilon}
\end{aligned}
$$

Hence the phase slip effect occurs as a fix offset, which value is $\lambda\left(E_{j}\right)^{\dagger} \nabla \Delta \phi_{\epsilon}$, where the phase slip error in the measurement is $\nabla \Delta \phi_{\epsilon}$.

\subsection{Multiple antenna based integer ambiguity resolution}

The orientation determination solution used multiple antennas on the board of the vehicle. This fact can be exploited in the position estimation solution as well. Consider the double differenced equation for $x_{b 1}, x_{b 2}$ and $x_{12}$

$$
\begin{aligned}
& \nabla \Delta \phi_{b 1, k}=\lambda^{-1} E_{k} x_{b 1, k}+\nabla \Delta N_{b 1}+\nabla \Delta \mu_{b 1, k} \\
& \nabla \Delta \phi_{b 2, k}=\lambda^{-1} E_{k} x_{b 2, k}+\nabla \Delta N_{b 2}+\nabla \Delta \mu_{b 2, k} \\
& \nabla \Delta \phi_{12, k}=\lambda^{-1} E_{k} x_{12, k}+\nabla \Delta N_{12}+\nabla \Delta \mu_{12, k}
\end{aligned}
$$

Using that $x_{b 2}=x_{b 1}+x_{12}$ and applying the definition of the double differenced measurement in (6) and (13) it can be proven that

$$
\nabla \Delta N_{b 2}=\nabla \Delta N_{b 1}+\nabla \Delta N_{12}
$$

where $\nabla \Delta N_{12}$ is already known from the orientation solution.

There are common solutions for the integer least squares problem given in (63) and (64). The LAMBDA and the MILES methods give suboptimal solutions for the problem. Their common property is that they are search space methods and they give more than one possible solution vector together with a possibility value for each solution. The inputs of these algorithms are an approximated solution for the double differenced integer ambiguity vector, called float point solution, and a covariance matrix characterizing the error of the approximation.

The algorithm for the precise position determination contains three steps. In the first step the integer ambiguity is determined separately for $x_{b 1}$ and $x_{b 2}$ using the LAMBDA or the MILES method (the authors used LAMBDA). For both cases a limited number of integer ambiguity vectors is selected (called integer ambiguity set). The authors used 30 possible integer ambiguity vectors in each set.
In the second step a search should be started for all pairs of integer ambiguity vectors from these sets and the search criteria is to satisfy 66. If more than one pair of integer ambiguity vector satisfy the criteria then that one is selected, which has the highest combined possibility. If there is no satisfying pair in the possible solutions, then the method fails and it should be repeated in the next measurement epoch.

The third step is the determination of the baselines $x_{b 1}$ and $x_{b 2}$ using the LS solution of (63)- 64)

$$
\begin{aligned}
& x_{b 1, k}=\lambda\left(E_{k}\right)^{\dagger}\left(\nabla \Delta \phi_{b 1, k}+\nabla \Delta N_{b 1}\right) \\
& x_{b 2, k}=\lambda\left(E_{k}\right)^{\dagger}\left(\nabla \Delta \phi_{b 2, k}+\nabla \Delta N_{b 2}\right)
\end{aligned}
$$

\subsection{Initialization of the LAMBDA method}

The integer least square solutions require an approximated integer ambiguity and a covariance matrix as input. Our covariance matrix determination method is based on the single receiver position determination algorithm, described in [10], where the base equation is

$$
\hat{\rho}^{i}=\sqrt{\left(x_{i}-X\right)^{2}+\left(y_{i}-Y\right)^{2}+\left(z_{i}-Z\right)^{2}}+C_{b}
$$

where $\hat{\rho}^{i}$ is the pseudorange measurement, $x_{i}, y_{i}, z_{i}$ are the components of the satellite positions, $X, Y, Z, C_{b}$ are the unknown user position and the clock bias, respectively. Forming the $\hat{\rho}^{i}$ measurements into a $\hat{\rho}$ vector, a non-linear equation $\hat{\rho}=h(\xi)$ can be formed, where the $\xi$ parameter vector consists of the unknown $X, Y, Z, C_{b}$ components. Introducing the residuals

$$
\begin{aligned}
\delta \xi & =\xi-\xi_{\text {nom }} \\
\delta \hat{\rho} & =h(\xi)-h\left(\xi_{\text {nom }}\right) \\
H & =\left.\frac{\partial h(\xi)}{\partial \xi}\right|_{\xi=\xi_{\text {nom }}}
\end{aligned}
$$

an iterative algorithm can solve the problem, using the solution of the

$$
\delta \hat{\rho}=H \delta \xi
$$

linear equation in least square sense.

Let $p_{b}$ be the known position of the base station and $p_{1}, p_{2}$ are the results of pseudorange based calculation (69)-(73). Then an approximated floating point solution for the double differenced integer ambiguity is

$$
\begin{aligned}
& \nabla \Delta \tilde{N}_{b 1}=\nabla \Delta \phi_{b 1, k}-\lambda^{-1} E_{k}\left(p_{1}-p_{b}\right) \\
& \nabla \Delta \tilde{N}_{b 2}=\nabla \Delta \phi_{b 2, k}-\lambda^{-1} E_{k}\left(p_{2}-p_{b}\right)
\end{aligned}
$$

As it is shown in [10] the covariance matrix of the $\delta \xi$ residual is

$$
E\left\langle\delta \xi(\delta \xi)^{T}\right\rangle=\sigma^{2}\left(H^{T} H\right)^{-1}
$$

where $\sigma$ is the standard deviation of the pseudorange measurement and $E\langle\rangle$ is the notation of the expected value. Let $R_{\rho}$ be 
the upper-left $3 \times 3$ submatrix of $\sigma^{2}\left(H^{T} H\right)^{-1}$, describing the covariance of the position measurement.

This covariance matrix can be used to form the covariance matrix of the uncertainty of the floating point integer ambiguity solution in the form of

$$
R_{\phi}=\lambda^{-1} E_{k} R_{\rho}
$$

It should be noted that the GPS receivers produce the components of the $E\left\langle\delta \xi(\delta \xi)^{T}\right\rangle$ in the form of horizontal dilution of precision (HDOP) and vertical dilution of precision (VDOP) [10], defined as

$$
\begin{aligned}
& H D O P=\sqrt{\operatorname{trace}\left(R_{\rho, 2 \times 2}\right)} \\
& V D O P=R_{\rho, 3,3}
\end{aligned}
$$

where $R_{\rho, 2 \times 2}$ is the upper-left $2 \times 2$ submatrix of $R_{\rho}$ and $R_{\rho, 3,3}$ is the lower right component of $R_{\rho}$. If the computation capacity of the algorithm is important, then the position output of the GPS receiver can be used as approximated position and the calculation of the matrix $H$ can be avoided by using an approximation for $R_{\rho}$ :

$$
\hat{R}_{\rho}=\text { blockdiag }\left(\frac{H D O P^{2}}{2}, \frac{H D O P^{2}}{2}, V D O P^{2}\right)
$$

\section{Precise position estimation}

The result of the orientation determination in section 3.5.4 is the $R_{B, N E D, k}$ orientation matrix. The angular velocity sensor and the magnetometer was used frequently in the estimation. The accelerometer measurement was required only in stationary situation during the initialization. Therefore it was enough that these sensors measure in a vehicle fixed coordinate frame, whose axes are parallel to the $K_{B}$ frame's axes.

During the position estimation, the accelerometer sensor will also be frequently used, therefore its position should be known in $K_{B}$. Let $p_{I M U}$ be this position. Let the position of the first and second GPS receivers be denoted by $p_{1, B}$ and $p_{2, B}$ respectively. The goal of the method is to define the position and the velocity of the origin of $K_{B}$, these variables are denoted by $p_{N E D}$ and $v_{N E D}$. Vector $p_{N E D}$ is defined relative to the position of the base station. The estimation is based on extended Kalman filter.

\subsection{Kinematic model formulation}

The continuous time kinematic model of a moving vehicle is

$$
\begin{aligned}
\dot{v}_{I M U, N E D} & =R_{B, N E D}^{T}\left(a+a_{b}\right)-\left[\begin{array}{c}
0 \\
0 \\
-g
\end{array}\right]+v_{a} \\
\dot{a}_{b} & =v_{b} \\
\dot{p}_{N E D} & =v_{I M U, N E D}-R_{B, N E D}^{T}\left(p_{I M U} \times \omega\right)+v_{v}
\end{aligned}
$$

where $v_{I M U, N E D}$ is the velocity of the accelerometer sensor in $K_{N E D}, a$ is the measured acceleration, $a_{b}$ is the accelerometer offset, $g$ is the gravitational acceleration and $\omega$ is the offset compensated angular velocity. These equations form a nonlinear relationship in the form of $\dot{x}=f_{\text {pos }}(x, u, v)$ using the notations

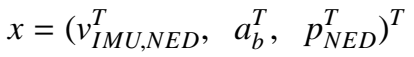

$$
\begin{aligned}
& u=a ; \quad v=\left(v_{a}^{T}, \quad v_{b}^{T}, \quad v_{v}^{T}\right)^{T}
\end{aligned}
$$

The connection between the kinematic model and the GPS based measurement can be described in two ways, depending on the capability of the GPS receiver.

Option 1 The simpler solution is when the GPS receiver does not provide Doppler measurements. In this case only the double differenced phase measurement is available. This can be used in the form of

$$
\begin{aligned}
& \nabla \Delta \phi_{b 1, k}=\lambda^{-1} E_{k} R_{E C E F, N E D}\left(p_{N E D, k}+p_{1, B}\right)+\nabla \Delta N_{b 1}+z_{1} \\
& \nabla \Delta \phi_{b 2, k}=\lambda^{-1} E_{k} R_{E C E F, N E D}\left(p_{N E D, k}+p_{2, B}\right)+\nabla \Delta N_{b 2}+z_{2}
\end{aligned}
$$

Option 2 If the receiver is able to provide precise Doppler measurements, then they can also be used in the state estimation. A Doppler like measurement is already introduced as $\delta \nabla \Delta \phi$ in 41]. Let the double differenced Doppler measurement be denoted by $\nabla \Delta D$ and the approximation relative to $\delta \nabla \Delta \phi$ is

$$
\nabla \Delta D \approx \frac{\delta \nabla \Delta \phi}{T_{s}}
$$

Its connection to the kinematic model in discrete time is

$$
\nabla \Delta D_{b 1, k}=\lambda^{-1} E_{k} R_{E C E F, N E D}\left(\left(p_{1, B}-p_{I M U}\right) \times \omega+v_{I M U}\right)+z_{3}
$$

$\nabla \Delta D_{b 2, k}=\lambda^{-1} E_{k} R_{E C E F, N E D}\left(\left(p_{2, B}-p_{I M U}\right) \times \omega+v_{I M U}\right)+z_{4}$

Option 1 alone or together with option 2 can form an equation $y=g_{\text {pos }}(x, z)$, where $y$ contains the GPS measurements and $z$ is the measurement noise vector.

\subsection{State estimation}

The first step is the discretization of the continuous time model. The authors used the Euler method. Let the discrete system be in the form of

$$
\begin{aligned}
x_{k+1} & =f_{\text {pos }, d}\left(x_{k}, u_{k}, v_{k}\right) \\
y_{k} & =g_{\text {pos }, d}\left(x_{k}, z_{k}\right)
\end{aligned}
$$

The state estimation is based on the extended Kalman filtering method using the algorithm in [10]. Using the following definition:

$$
\begin{aligned}
A_{k-1} & =\left.\frac{\partial f_{p o s, d}}{\partial x}\right|_{\hat{x}_{k-1}} \\
C_{k} & =\left.\frac{\partial g_{p o s, d}}{\partial x}\right|_{\bar{x}_{k}},
\end{aligned}
$$

the estimation algorithm is 
Time update

$$
\begin{aligned}
\bar{x}_{k} & =f_{p o s, d}\left(\hat{x}_{k-1}, u_{k-1}, 0\right) \\
M_{k} & =A_{k-1} \Sigma_{k-1} A_{k-1}^{T}+R_{v, k-1} \\
G_{k} & =M_{k} C_{k}^{T}\left(C_{k} M_{k} C_{k}^{T}+R_{z, k}\right)^{-1} \\
\Sigma_{k} & =M_{k}+G_{k} C_{k} M_{k}
\end{aligned}
$$

\section{Measurement update}

$$
\hat{x}_{k}=\bar{x}_{k}-G_{k}\left(y_{k}-g_{p o s, d}\left(\bar{x}_{k}, 0\right)\right)
$$

\subsection{Determination of the covariance matrix}

It is essential to give a good estimation of the covariance matrices in the Kalman filter, therefore the following method is introduced in our system. Two covariance matrices appear in the extended Kalman filter algorithm. The first is $R_{v, k}$ and corresponds to the state vector noise and the second $R_{z, k}$ refers to the measurement noise. The values of $R_{v, k}$ can be originated from the noise of the accelerometer. Let $R_{a}$ be the covariance matrix of the accelerometer. This can be obtained by the analysis of the measurements in stationary situation.

It is hard to determine the $\sigma$ variance of the noise of the offset (bias) part of the state vector, denoted by $R_{b}$. In practice, good result can be achieved, when its elements are one order less than the elements of $R_{a}$.

The last part of the state vector covariance matrix corresponds to the velocity of the vehicle. Its value in the kinematic model is determined by the integration of the acceleration. Therefore an appropriate choice for the $R_{v}$ covariance matrix can be $R_{v}=$ $T_{s} R_{a}$.

Assuming that the three parts of the state vector are independent, the $R_{v}$ matrix is

$$
R_{v}=\operatorname{blockdiag}\left(R_{a}, R_{b}, R_{v}\right)
$$

The measurement covariance matrix can be originated from the carrier phase and Doppler measurements. The size of $R_{z}$ is $2(m-1) \times 2(m-1)$ in the case of option 1 and $4(m-1) \times 4(m-1)$ in case of option 2. Let the covariance matrix of $\nabla \Delta \phi$ be $R_{\phi}$ and the covariance matrix of $\nabla \Delta D$ be $R_{D}$, then

$$
R_{z}=\operatorname{blockdiag}\left(R_{\phi, b 1}, \quad R_{\phi, b 2}, \quad R_{D, b 1}, \quad R_{D, b 2}\right)
$$

To determine $R_{\phi}$ the following method can be used. The vehicle should stay in a fix position and orientation. The integer ambiguity and the position can be determined in this situation using the position determination method in Section 4 . Then $\nabla \Delta N$ and $x_{k}$ is known. The error of the carrier phase measurement is

$$
\epsilon_{\phi, k}=\nabla \Delta \phi_{k}-\nabla \Delta N-\lambda^{-1} E_{k} x_{k}
$$

then analyzing the measured values the covariance matrix yields

$$
R_{\phi}=E\left\langle\epsilon_{\phi, k} \epsilon_{\phi, k}^{T}\right\rangle
$$

A similar method can be used to determine $R_{D}$.

\section{System realization and experimental results}

During our research a prototype of a data acquisition and signal processing system was developed. The accuracy and the reliability of the system are tested during car movement tests. One of the future applications of the system will be a project where the task is the identification of the nonlinear dynamic model of an airplane based on the estimated states.

\subsection{System realization}

The structure of the onboard system can be seen in Fig. 7. The GPS receivers are Ublox LEA-6T modules, with 5Hz sampling rate and carrier cycle measurement capability. They are also able to produce a precise GPS clock synchronized time pulse. This pulse is the base of the time synchronization of the whole system. An Atmel AT91SAM7A3 processor is responsible for the synchronized data collection of the sensors. The accelerometer module has an Analog Devices ADXL330 3D acceleration sensor and the angular velocity sensor is based on three pieces of Analog Devices ADXRS613. The analog outputs of the accelerometer and the angular velocity sensors are sampled by 24 bit analog-digital converters. The magnetic sensor is a Freescale MAG3110 module. The inertial and magnetic sensors are calibrated using the methods of [12] and [13].

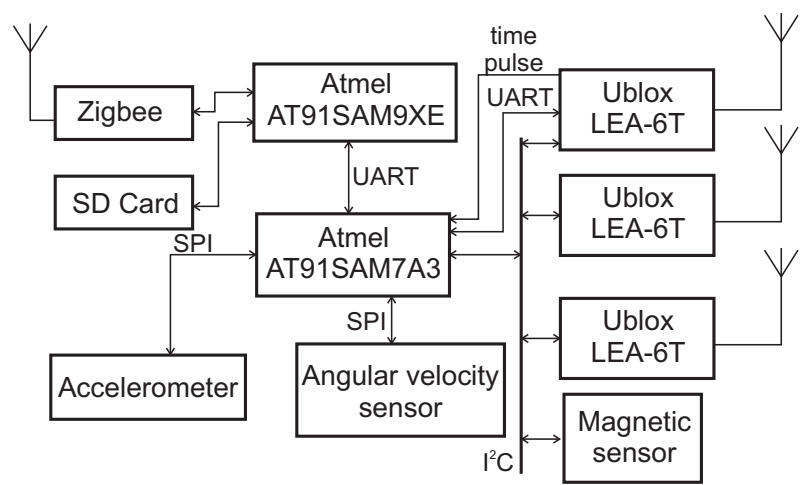

Fig. 7. Structure of the onboard data acquisition system

The data procession of the onboard system is running on an Atmel AT91SAM9XE 200MHz fixed point processor using a soft real-time Linux operating system. An XBee Pro Series 2 module is used for real time communication which is able to operate in a Zigbee communication network. This module can easily be replaced with a GSM module in the further development.

The structure of the base station can be seen in Fig. 8 The center of the station is an Olimex L9260 board, containing an Atmel AT91SAM9XE processor. This board communicates with the Ublox LEA-6T GPS receiver and transmits data to the moving vehicle by an Xbee module. Both the onboard module and the base station are logging the measurement data to SD cards. Therefore offline procession of the data is also possible.

\subsection{Real-time experiments}

First test: simple car motion In our first test the orientation and position determination methods are tested during a planar 


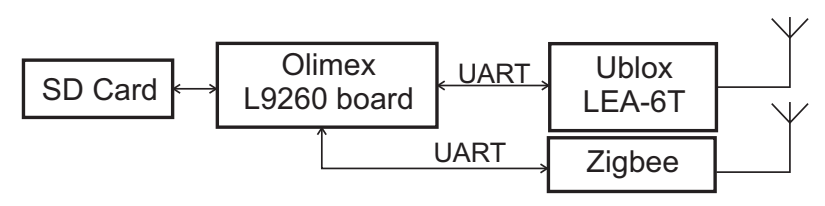

Fig. 8. Structure of the base station

movement of a car. The path of the car can be seen in Fig. 9 The duration of the movement was two and a half minutes. The measurements were taken in an urban area.

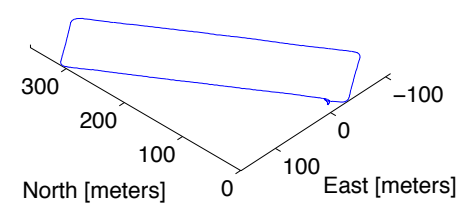

Fig. 9. Path of the car

First the orientation estimation was performed on the measured data set. The results can be seen in Fig. 10 and in Fig. 11. During the car movement it is hard to determine the real orientation to produce information to which the calculated results can be compared. To overcome the problem car motion consisting of straight line sections in horizontal plane were chosen for which the error can be easily detected. In this case an approximated orientation was also calculated using the measurements from the magnetometer.
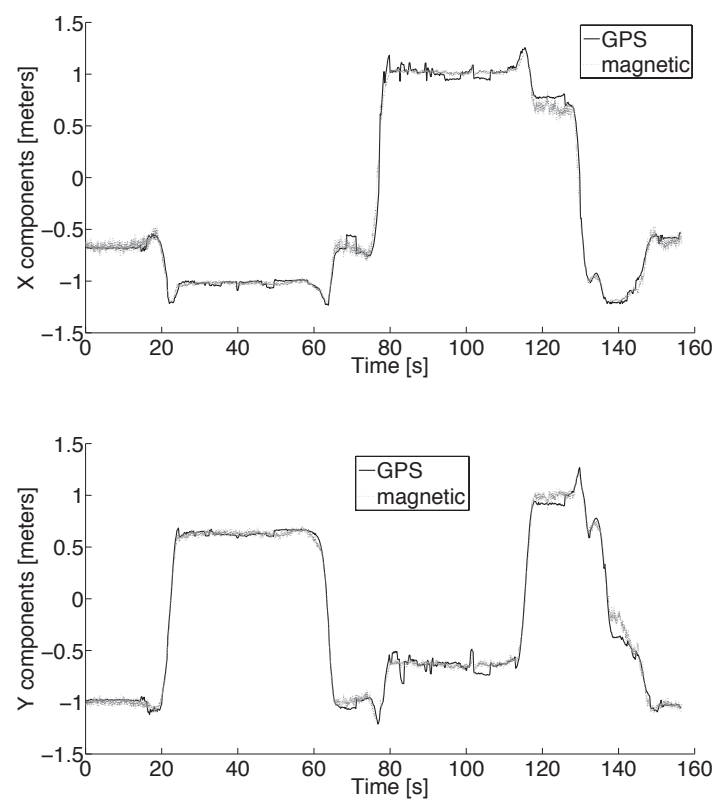

Fig. 10. Components of the calculated baseline, $x_{12}$

It can be seen in Fig. 10, that in some cases the GPS based calculation gives results which are different from the reference value. The largest amount of error occurs after the $80^{\text {th }}$ second. The source of errors is the frequent change of the satellites. The number of the available satellites can be seen in Fig. 12 Comparing Fig. 10 and Fig. 12, it can be seen that larger errors occur, as the number of satellites is 4 or less. If there are 4 visible satellite, $E_{k}$ is quadratic and the baseline determination problem is not over-determined. This means that the result is sensitive for an error in the carrier phase measurement. If the number of available satellites is below 4 , the dead reckoning is in operation.

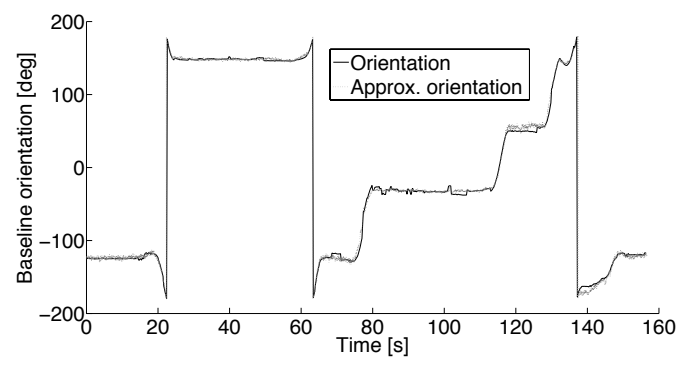

Fig. 11. Orientation relative to the North direction

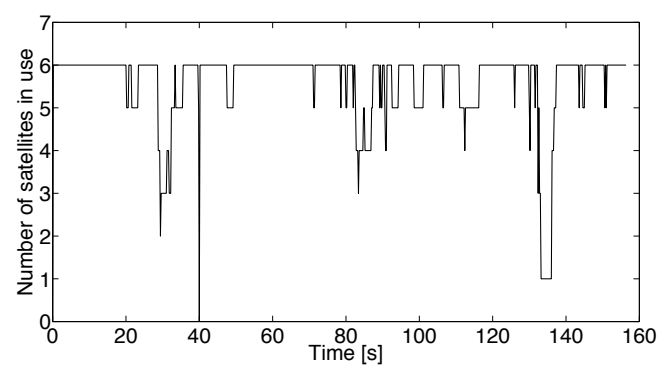

Fig. 12. Number of satellite in use during orientation estimation

Examining the measurements shows that before the receiver loses the lock of a satellite, the signal to noise ratio decreases. This phenomenon causes the errors in the measurements. It can also be seen that when a larger number of receivers is available again, the calculated values are close to the reference. In Fig. 11 it can be seen that even in these situations, the error in the orientation is less than 5 degree which means that the orientation of the car is still reliable.

It should be noted that in this test the considered number of satellites is restricted to 6 to meet the real time computation capacity of perspective applications. In every epoch those 6 satellites were chosen, that had the highest elevation angle. It should be noted that these satellites were those 6 which have the best SNR values.

One way to verify the reliability of the method is to examine the measured length of $x_{12}$. As receiver 1 and 2 are fixed to the roof of the car, it is known that the real baseline length is 1.21 meters. The measured values are shown on Fig. 13 .

The result of the position estimation can be seen in Fig. 14 The detailed zoom of the lower part of Fig. 14 shows the effectiveness of the state estimation. The GPS measurements have $200 \mathrm{~ms}$ sampling time and the IMU measured with $10 \mathrm{~ms}$ sampling time. This means that between two consecutive GPS measurements only the time update part of the Kalman filter (95)988 was used. Hence during $200 \mathrm{~ms}$, the state estimation is based on only the IMU. It can be seen in Fig. 14 that at the end of every $200 \mathrm{~ms}$ long period, the estimated position changes smoothly after every GPS measurements. 


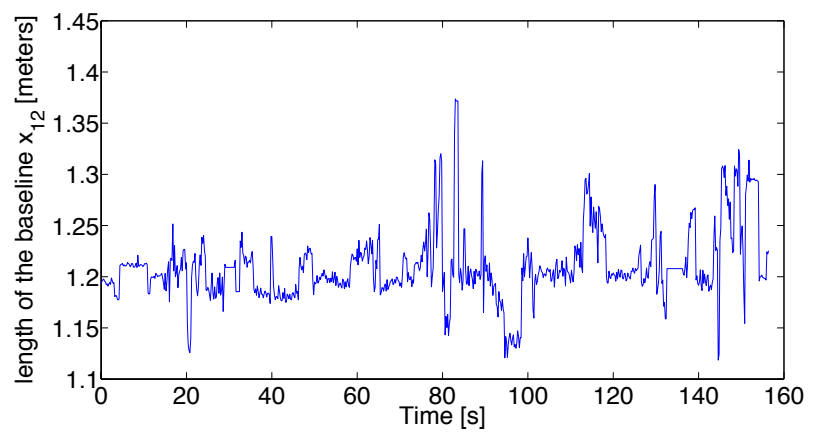

Fig. 13. The measured value of $x_{12}$ during car movement

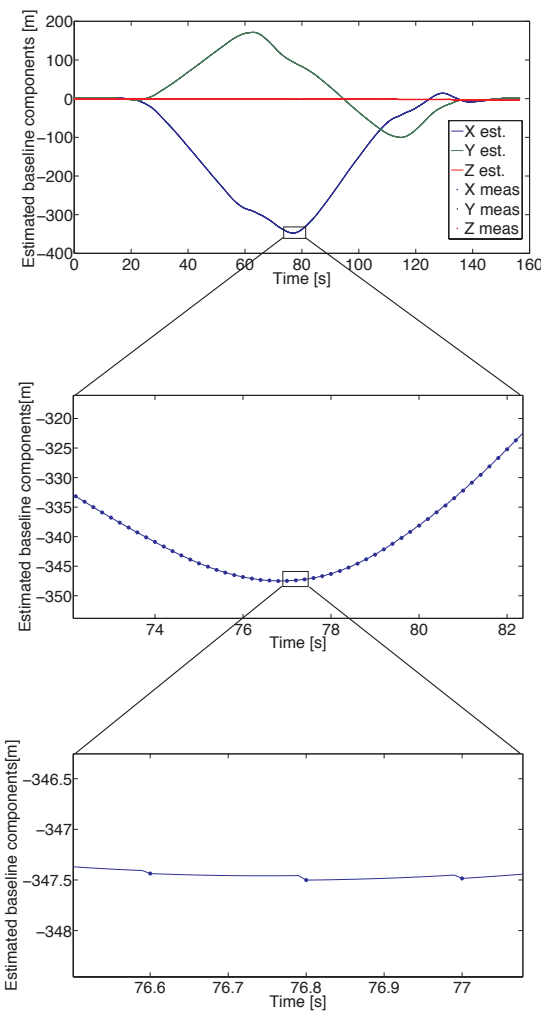

Fig. 14. The estimated position and a detailed zoom which shows the smooth result between the GPS measurements

During this test the Option 1 solution of Section 5 was used. The covariance matrix determination is the method in Section 5.3 The variances along the axes of the accelerometer are calculated from stationary measurements. The results are $\sigma_{X}=0.00096 \frac{m^{2}}{s^{4}}, \sigma_{Y}=0.00103 \frac{m^{2}}{s^{4}}, \sigma_{Z}=0.0011 \frac{m^{2}}{s^{4}}$. Hence a practical choice for $R_{a}$ is $R_{a}=\operatorname{diag}\left(\left[10^{-3}, 10^{-3}, 10^{-3}\right]\right)$. Based on Section $5.3 R_{b}=\operatorname{diag}\left(\left[10^{-4}, 10^{-4}, 10^{-4}\right]\right)$ and $R_{v}=\operatorname{diag}\left(\left[10^{-5}, 10^{-5}, 10^{-5}\right]\right)$.

The variance of a single channel carrier phase observable is measured in stationary state. The result is $\sigma_{\phi}=0.89 \cdot 10^{-4}$. The choice for $R_{\phi}$ was $R_{\phi}=\operatorname{diag}\left(\left[10^{-4}, \ldots, 10^{-4}\right]\right)$.

The error of the position estimation can be formed as

$$
\epsilon_{k, e s t}=\hat{x}_{k, 7: 9}-x_{b 1, k}
$$

where $\hat{x}_{k, 7: 9}$ is the estimated position elements of 84 and $x_{b 1, k}$ is the precise position measurement. This error value can be

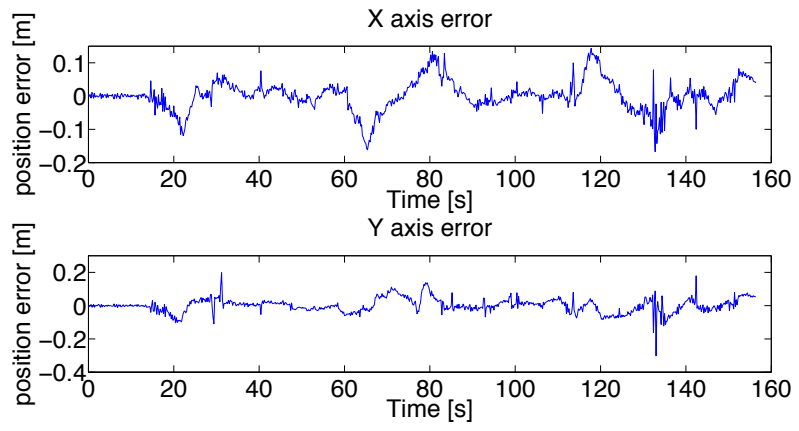

Fig. 15. Position estimation error during car movement

calculated when $x_{b 1, k}$ is available. The horizontal error is shown in Fig. 15

Second test: complex car motion The second test involves a more complex planar movement with car in an urban area. It already contains more turns than the first test. In this case the availability of the GPS signals was also restricted, $20.5 \%$ of the whole time of the experiment should be navigated based only on the IMU. The path of the movement can be seen in Fig. 16.

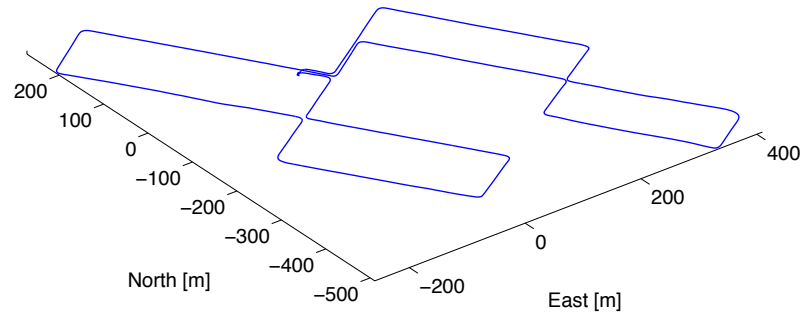

Fig. 16. Path of a complex urban area movement

If the coordinate frame of the car is defined as on Fig. 3 then the $\mathrm{X}$ axis of $K_{B}$ is the heading direction as well. The slipping angle of a car is defined as the angle between the heading direction and the direction of the velocity. If the car movement is planar and straight, the side slip angle is negligible [14].

The place of the second experiment is chosen as it contains straight line segments. The velocity of the car is a direct output from the Ublox LEA6-T GPS receiver. During the car movement this information is collected and the direction of the velocity is used as a reference in the actual test. The resulted orientation and the difference from the reference value is shown in Fig. 17.

Fig. 17presents also the time periods when the GPS signals are unavailable. One can see that this situation can occur frequently in an urban area. After each dead-reckoning period the recalculation of the integer ambiguities are necessary. Therefore it is important that our algorithm can determine the ambiguities from a single measurement epoch within 0.2 seconds.

The length of the baseline is also compered to the real value. This length is shown in Fig. 18. In this experiment the vector $x_{12}$ was 1.18 meters long. 

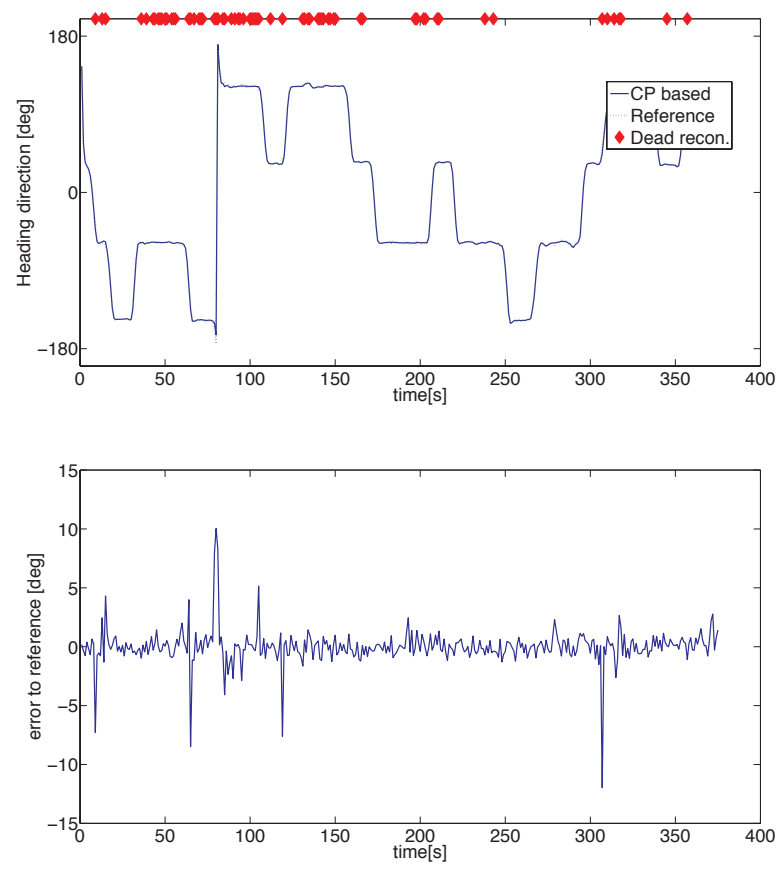

Fig. 17. Heading direction of a car, and the error to the reference

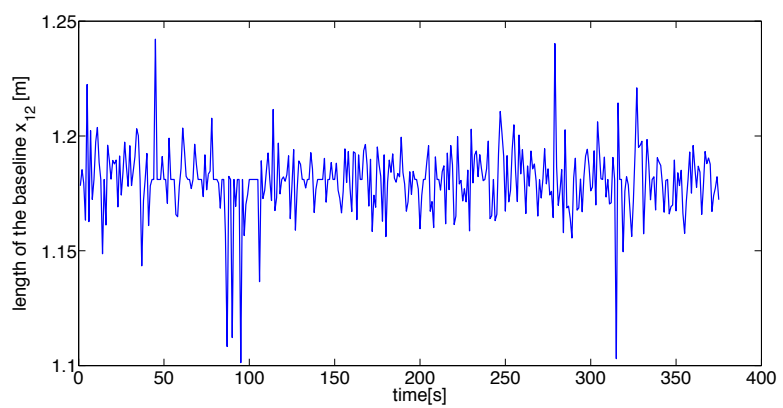

Fig. 18. The length of $x_{12}$ during the second test

Third test: long time car motion The third experiment verifies the long time reliability of the system. In this case typical traffic situations were tested during an about $110 \mathrm{~km}$ and 100 minutes long movement. The path contained crowded urban areas, smooth maneuvers in traffic, hilly areas (non-planar movement) and high speed road movements as well. The speed profile of the movement can be seen in Fig. 19. In this case $30.9 \%$ of the whole time was in a GPS denied area.

During the test the length of $x_{12}$ was measured and compared to the real 1.18 meters value. The measurement result can be seen in Fig. 20.

Forth test: airplane motion The next experiment is connected to one of the future applications of our system. The future task is to determine the nonlinear dynamic model of a sail-plane. One of the first steps of this project is the determination of the position and the orientation of the aircraft.

In this test our system was attached to the body of a sail-plane and a low-altitude flight was performed. The path of the plane can be seen in Fig. 21 .

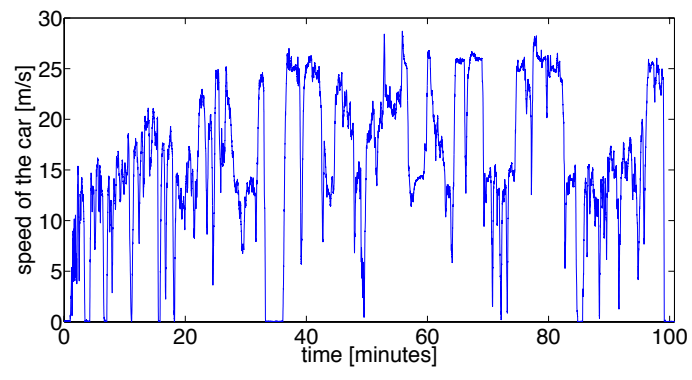

Fig. 19. Speed of a car during the long time test

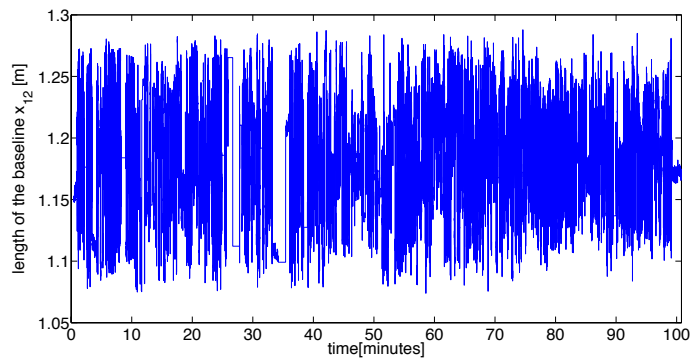

Fig. 20. Length of $x_{12}$ during the long time measurement

After calculating $R_{B, N E D}$, the orientation is represented by RPY angles on Fig. 22 .

\section{Conclusion}

This paper presents a solution to precise GPS based orientation and position determination for moving vehicles. The main problem of the carrier phase based GPS methods is the integer ambiguity determination. In the case of the orientation determination, this problem is solved using additional sensors of the vehicle. On the board of the moving body three GPS antennas are present. If the integer ambiguity was determined, the orientation of the vehicle can be calculated. An iterative approach was presented for solving the integer ambiguity problem, which meets the requirements of real-time expectations.

In the case of the position determination it is proven that the inertial sensors have no additional information for the integer ambiguity solution. The method presented here uses the classical LAMBDA algorithm to solve the integer least squares problem, but advantage is taken of the multiple receivers on the moving body. This way, a more reliable solution has been obtained for the integer ambiguities.

For each of the orientation and position parts of the calculations, extended Kalman filter based state estimators have been used to smooth the GPS based measurements and to provide sensor fusion based on measurements of the inertial sensors and GPS. The paper also presents the low-cost hardware/software architecture of the navigation system. The presented methods are tested in real environment.

The advantage of the algorithms is that the integer ambiguity can be resolved using measurements from only one epoch. This means that the solutions can be initialized fast and are capable of switching back from dead reckoning to normal operation very quickly. 


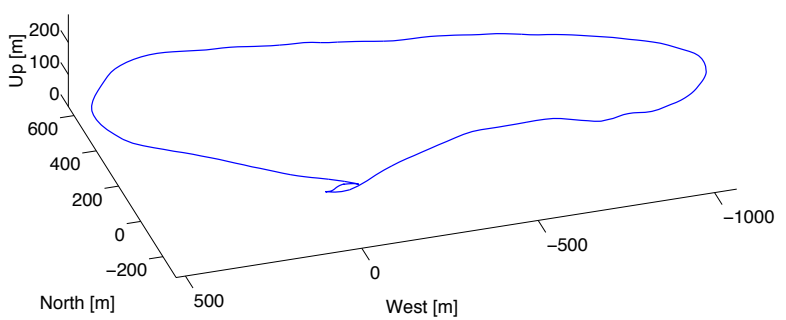

Fig. 21. Path of the sail-plane

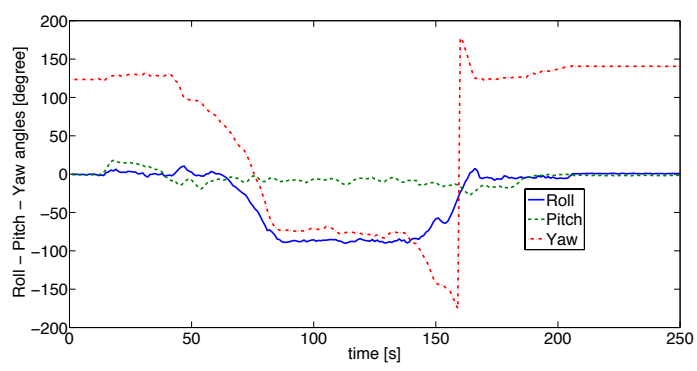

Fig. 22. RPY orientation of a sail-plane during the flight

\subsection{Future work}

The presented experimental results show the effectiveness of the developed methods, however sometimes measurements with larger error occur, especially when the number of the available satellites is low. Methods are needed to identify these situations and further improve the properties of the navigation system. Important new research direction is the estimation of the angle of attack and the sideslip angle of aerial vehicles and their correction by the wind effect.

\section{Appendices}

\section{A. The carrier phase observables}

This article uses GPS receiver which has L1 frequency measurements only. In this case the nominal frequency of the incoming signal is $f_{N}=1575.42 \mathrm{MHz}$. Because of the movement of the satellite and the receiver the measured frequency is affected by the Doppler effect. The difference between the measured and the nominal frequency is called Doppler frequency shift [11] and denoted by $f_{D}$.

The carrier cycle measured by receiver $r$ for the $i^{t h}$ satellite signal is

$$
\phi_{r}^{i}(t)=\phi_{i n i t, r}^{i}+\int_{t_{0}}^{t} f_{D}(\tau) d \tau
$$

where $\phi_{r}^{i}(t)$ is the measurement output of the receiver, $\phi_{\text {init, } r}^{i}$ is a random integer number relating to the initialization of the phase locked loops (PLLs) of the receiver and $t_{0}$ is the time instant when the PLLs lock the incoming signal. The measurement output is assumed to be in wavelength unit.

\section{B. The integer ambiguity problem}

The integer ambiguity problem is originated from the fact that two carrier waves cannot be distinguished from each other by the receiver. Therefore an integer number of wavelength ambiguity appears in the distance measurement between the receiver and the satellites. This problem leads to the integer least squares problem, whose mathematical form is

$$
y=A x+B N \quad x \in \mathbb{R}^{3} \quad N \in \mathbb{Z}^{m-1}
$$

where $x$ is an unknown position vector, $N$ is an unknown vector of integers called integer ambiguity, $y$ is a measurement vector from the GPS receiver, $m$ is the number of visible satellites and $A$ and $B$ are known real matrices, respectively. Equation 18 in Section 2 belongs to this type of mathematical problems.

There are some common suboptimal solutions for this problem, the popular ones are the LAMBDA method [17] and the (modified) MLAMBDA method [4]. There are some practical problems with the LAMBDA based methods. They need some minutes if the set of the visible satellites is changing (reinitialization) [3]. They also require the knowledge of the correlation matrix of the carrier phase measurements.

Although there are also methods which can determine the integer ambiguity from a single measurement, but in this case the number of false positive results is high [15].

\section{References}

1 Navstar GPS Space Segment Navigation User Interfaces, 1997, Standard No.:Drawing number:ICD-GPS-200.

2 Bell T, Automatic tractor guidance using carrier-phase differential GPS, Computers and Electronics in Agriculture, 25(1-2), (2000), 53-66, DOI 10.1016/S0168-1699(99)00055-1

3 Chang X-W, Paige C, Yin L, Code and carrier phase based short baseline GPS positioning: computational aspects, GPS Solutions, 7(4), (2004), 230240, DOI 10.1007/s10291-003-0077-z

4 Chang X-W, Yang X, Zhou T, MLAMBDA: a modified LAMBDA method for integer least-squares estimation, Journal of Geodesy, 79(9), (2005), 552 565, DOI 10.1007/s00190-005-0004-x

5 Chang X-W, Xie X, Zhou T, MILES: MATLAB package for solving Mixed Integer LEast Squares problems, Version 2.0, Scientific Computing Laboratory, School of Computer Science, McGill University, October 2011.

6 Farrell J, Barth M, The Global Positioning System and Inertial Navigation, McGraw-Hill, 1998.

7 Farrell J, Givargis T, Barth M, Real-time differential carrier phase GPSaided INS, IEEE Transactions on Control Systems Technology, 8(4), (2000), 709-721, DOI $10.1109 / 87.852915$

8 Farrell J, Aided Navigation - GPS with High Rate Sensors, McGraw-Hill, 2008.

9 Fischler M, Bolles R, Random sample consensus: a paradigm for model fitting with applications to image analysis and automated cartography, Communications of the ACM, 24(6), (1981), 381-395, DOI $10.1145 / 358669.358692$

10 Grewal M, Weill L, Andrews A, Global Positioning Systems, Inertial Navigation, and Integration, Wiley, 2007, DOI 10.1002/0470099720

11 Kaplan E, Hegarty C, Understanding GPS - Principles and Applications, Artech House, 2005.

12 Kis L, Lantos B, Sensor fusion and actuator system of a quadrotor helicopter, Periodica Polytechnica Electrical Engineering, 53(3-4), (2009), 139150, DOI 10.3311/pp.ee.2009-3-4.06 
13 Kis L, Lantos B, GPS based attitude determination using embedded magnetometer calibration, Mediterranean Conference on Control and Automation, (Platanias-Chania, Greece, 2013), In:.

14 Lantos B, Márton L, Nonlinear Control of Vehicles and Robots, Advances in Industrial Control, Springer; London, 2011, DOI 10.1007/978-1-84996122-6

15 Li Y, Zhang K, Grenfell R, Improved Knight method based on narrowed search space for instantaneous GPS attitude determination, Navigation, $\mathbf{5 2}$, (2005), 111-119.

16 Sickle J, GPS for Land Surveyors, Ann Arbor Press, 2001.

17 Teunissen P, Least-square estimation of the integer GPS ambiguities.

18 Wang J, Satirapod C, Rizos C, Stochastic assessment of GPS carrier phase measurements for precise static relative positioning, Journal of Geodesy, 76(2), (2002), 95-104, DOI 10.1007/s00190-001-0225-6 